\title{
Preparation of Berbamine Loaded Chitosan-agarose Microspheres and in vitro Release Study
}

\author{
Zhang Hu, Sidong Li, Lei Yang \\ Department of Chemistry, College of Science, Guangdong Ocean University, China
}

\begin{abstract}
Berbamine loaded chitosan-agarose microspheres were prepared using a water-in-oil emulsion technique. Optimum preparing parameters were determined by orthogonal experiments as follows: ratio of berbamine to chitosan (w/w) is 1:10; percentage of emulsifier ( $\operatorname{span} 80, \mathrm{v} / \mathrm{v}$ ) is $6 \%$; volume of glutaraldehyde is $2 \mathrm{~mL}$; and reaction temperature is $70{ }^{\circ} \mathrm{C}$. Under these optimal conditions, the encapsulation efficiency and loading capacity of microspheres are $84.57 \%$ and $8.44 \%$, respectively. The swelling tests showed that the microspheres possessed higher swelling ratio at $\mathrm{pH} 7.4$ than at $\mathrm{pH}$ 1.2. FTIR indicated that berbamine had been successfully loaded in the chitosan-agarose microspheres by physical entrapment. In vitro release studies showed that berbamine was released from microspheres in a significantly sustained fashion.
\end{abstract}

Keywords: Chitosan, agarose, microspheres, berbamine.

\section{Introduction}

Berbamine, a natural compound from the plant Berberis amurensis, is a bis-benzylisoquinoline alkaloid and has been widely used in China for leukopenia treatment over the past decades. Clinical studies showed that berbamine could stimulate normal hematopoiesis of cancer patients undergoing chemotherapy or radiotherapy and has been used to protect tumor patients from cytotoxic effects of chemotherapeutic agents on bone marrow. Meanwhile, berbamine has anti-inflammatory, anti-arrhythmic effects and antineoplastic activity ${ }^{[1,2]}$. However, on oral administration, berbamine may produce some side effects, such as jaundice, stomach upset, lethargy, nose bleed, skin and eye irritation, kidney irritation, and so on. Therefore, it is essential to deliver berbamine at the intended therapeutic concentrations to the target sites to elicit its activity. Sustained delivery of berbamine may reduce the systemic side effects and provide effective and safe therapy of leukopenia that may reduce the dose and duration of therapy when compared with the conventional treatment.

Recently, microspheres have received great attention because of a variety of applications such as delivery vesicles for drugs, DNA, antigens, and protection proteins and enzymes, especially for controlled or sustained drug-delivering systems ${ }^{[3,4]}$. Generally, to make drugs encapsulated into microspheres may mask taste and odor, stabilize the quality of the drug, improve gastrointestinal tolerance and provide sustained release after oral administration. There has been considerable interest in developing microspheres prepared from chitosan matrices as effective drug delivery devices. Owing to its nontoxicity, biodegradability, biocompatiblity, mucoadhesion and antibacterial, chitosan, a natural polysaccharide, has been extensively applied in various areas, especially in the pharmaceutical and biomedical fields, such as drug delivery, wound dressing and antimicrobial agents ${ }^{[5,6]}$. It is reported that chitosan microspheres showed high cytotoxic activity toward tumor cells, while low toxicity against normal human liver cells ${ }^{[7]}$. The unique cationic character of chitosan microspheres could also provide higher affinity with negatively charged biological membranes and site-specific targets in vivo ${ }^{[8,9]}$. Therefore, chitosan microspheres were extensively used for drug delivery, such as oral administration to the colon, nasal delivery, transdermal delivery, parenteral delivery, implants and ocular delivery ${ }^{[6]}$. The introduction of a second ingredient into chitosan microspheres may increase their versatility in terms of the adsorption and delivery of some special drugs and their susceptibility to interact with biological surface. Agarose obtained from red seaweeds is a natural polysaccharide. It is of unique characteristic of forming a heat-reversible gel in dilute aqueous solution that is hydrophilic and macroporous. Agarose microspheres have been widely used in chromatographic separation, food industry, cell encapsulation and pharmacy as their mechanical properties and size can be readily controlled $^{[10-12]}$. To our knowledge, there are few reports that offer their investigations on chitosan-agarose microspheres ${ }^{[13]}$.

In this paper, the microspheres were prepared using the composite polymers, chitosan and agarose, and berbamine was chosen as a model drug to test the release behavior of the microspheres.

\section{Experimental}

\section{Materials and reagents}

Chitosan with a degree of deacetylation of $90 \%$ was purchased from Guoyao Biochemical Co. Ltd, Shanghai, China. Agarose was a gift from Taixing Bio-Tec Co., Ltd Lianjiang, China. Berbamine was purchased from Sichuan Shikun Medical Raw Materials Co., Ltd. Liquid paraffin, span 80, glutaraldehyde, acetone and acetic acid were of analytical grade.

\section{Preparation of microspheres}

The microspheres were prepared by water-in-oil (w/o) emulsion technique and were further utilized to encapsulate berbamine. Chitosan solutions were prepared by dissolving $1.5 \mathrm{~g}$ chitosan in $150 \mathrm{~mL}$ of $1 \%$ acetic acid to form $1 \%(\mathrm{w} / \mathrm{v})$ chitosan solutions. Agarose solutions were prepared by dissolving $0.75 \mathrm{~g}$ agarose powder in $150 \mathrm{~mL}$ distilled water to form $5 \%$ o $(\mathrm{w} / \mathrm{v})$ agarose solutions. Chitosan solutions were slowly added into the agarose solutions while stirring to form the aqueous solutions. Known weight of berbamine was completely dispersed in $50 \mathrm{~mL}$ 
of liquid paraffin under sonication as the oil phase. Span 80 was used as an emulsifier. A w/o emulsion was prepared by slowly adding the above-mentioned $30 \mathrm{~mL}$ aqueous solutions to the oil phase at a set temperature while being agitated by a homogenizer. Stirring was continued for 1 hour until a stable w/o emulsion was obtained. After addition of $25 \%(\mathrm{w} / \mathrm{w})$ aqueous glutaraldehyde as the crosslinking agent, the mixture was stirred for another 2 hours. The emulsion was quickly cooled to $30^{\circ} \mathrm{C}$ in a water bath allowing the formation of soft solid chitosan-agarose microspheres. The microspheres were collected by repeated washing, centrifugation and finally dried in a vacuum desiccator. The preparation process variables of microspheres were optimized by orthogonal experiment.

\section{Evaluation of drug loading efficiency}

$50 \mathrm{mg}$ microspheres were accurately weighed to the vial, and treated with ultrasonic at $60{ }^{\circ} \mathrm{C}$ for 3 hours in a water bath after the addition of $50 \mathrm{~mL}, 0.1 \mathrm{~mol} . \mathrm{L}^{-1}$ of $\mathrm{HCl}$ solution. The suspension was centrifuged, and then the supernatant was decanted and diluted appropriately. The amount of free drug in the supernatant was measured with a spectrophotometer at $282 \mathrm{~nm}$. The encapsulation efficiency (EE) and loading capacity (LC) of microspheres were calculated as follows:

$$
\mathrm{EE}=\frac{\mathrm{M}_{1}-\mathrm{M}_{2}}{\mathrm{M}_{1}} \times 100 \quad \mathrm{LC}=\frac{\mathrm{M}_{1}-\mathrm{M}_{2}}{\mathrm{M}_{3}} \times 100
$$

Where $M_{1}$ is the total amount of berbamine; $M_{2}$ is the amount of free berbamine; $M_{3}$ is the microspheres weight. All measurements are performed in triplicate.

\section{Swelling test}

$50 \mathrm{mg}$ dry microspheres encapsulated with berbamine were immersed in either phosphate buffer solution $(\mathrm{pH} 7.4)$ or hydrochloric acid solution $(0.1 \mathrm{M}, \mathrm{pH} 1.2)$ for a designated time at room temperature. The swollen samples were collected by centrifugation, and then carefully blotted with filter paper to remove the water on the surface, and immediately weighed. The swelling ratio was calculated using the following equation:

$$
\text { Swelling ratio } \%=\frac{\mathrm{W}_{\mathrm{t}}-\mathrm{Wd}_{\mathrm{d}}}{\mathrm{Wd}_{\mathrm{d}}} \times 100 \%
$$

Where $\mathrm{W}_{\mathrm{t}}$ and $\mathrm{W}_{\mathrm{d}}$ are the weights of the microspheres at the designated time swollen state and at the dry state, respectively.

\section{Fourier transform infrared spectroscopy (FTIR) analysis}

The interactions between drug and polymers were studied by FTIR PerkinElmer Spectrum 100 spectroscopy. Samples were prepared in $\mathrm{KBr}$ disks. The disks were obtained by compacting with a hydrostatic press at a force of 10 tons for 1 minute. The spectrum was recorded in the wave number range from 4000 to $450 \mathrm{~cm}^{-1}$ with a resolution of $4 \mathrm{~cm}^{-1}$ over 16 cumulative scans at room temperature.

\section{Evaluation of in vitro drug release}

Known amounts of the microspheres were suspended in phosphate buffer solution ( $\mathrm{pH} 7.4,20 \mathrm{~mL}$ ) and incubated at $37{ }^{\circ} \mathrm{C}$ under stirring. At varying time points, aliquots of sample were withdrawn from the receiver compartment. The supernatants were isolated by centrifugation and assayed to detect the drug release by UV spectroscopy at $282 \mathrm{~nm}$. After each sampling, the same volume of fresh phosphate buffer was added to the receiver compartment to maintain the constant volume.

\section{Results and Discussion}

\section{Optimization of microspheres preparation process}

Chitosan-agarose microspheres were prepared by w/o emulsification process. The optimum conditions for preparation of microspheres were investigated by independent variation of four parameters: A-ratio of berbamine to chitosan (w/w), B-percentage of emulsifier (span 80, v/v), C-volume of glutaraldehyde (mL) and D-reaction temperature. For each parameter, the three values were investigated. The investigated variables and their test levels were listed in Table 1. Reference to the experimental design theory, the orthogonal array L9 $\left(3^{4}\right)$ was selected to arrange the test program. The encapsulation efficiency (EE) and loading capacity (LC) were designated as the quality indexes (QI). The test results were shown in Table 2.

As shown in Table 2, volume of glutaraldehyde was the most important factor to the microspheres preparation among the four selected factors according to $\mathrm{R}$ value. The importance of four factors to the microspheres preparation is in a sequence from high to low as follows: volume of glutaraldehyde, reaction temperature, ratio of berbamine to chitosan, percentage of emulsifier. Obviously, the optimum level of each variable is A-2, B-3, C-2, and D-2 according to the mean value. Thus the optimum reaction conditions were as follows: ratio of berbamine to chitosan (w/w), 1:10; percentage of emulsifier (span 80, v/v), 6\%; volume of glutaraldehyde, $2 \mathrm{~mL}$; and reaction temperature, $70{ }^{\circ} \mathrm{C}$. Under these optimal conditions, the encapsulation efficiency and loading capacity of microspheres are $84.57 \%$ and $8.44 \%$, respectively.

\section{Swelling ratio}

The swelling kinetics and time dependent swelling behaviours of chitosan-agarose microspheres in phosphate buffer solution ( $\mathrm{pH}$ 7.4) and hydrochloric acid solution (0.1 M, pH 1.2) at room temperature are given in Figure 1. The microspheres showed higher swelling ratio in phosphate buffer solution $(\mathrm{pH} 7.4)$ than in an acid condition ( $\mathrm{pH}$ 1.2). This can be explained that the Schiff's base

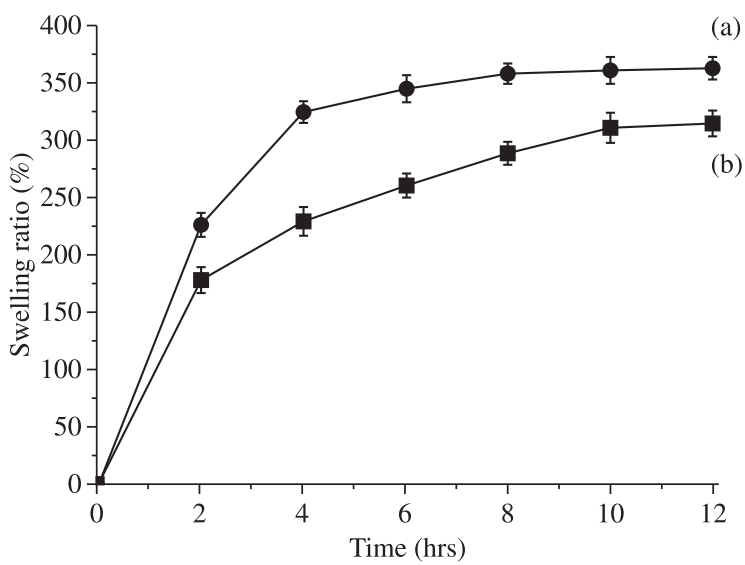

Figure 1. Swelling behaviour of the microspheres. a) at $\mathrm{pH} 7.4$; b) at $\mathrm{pH} 1.2$.

Table 1. The investigated variables and their levels.

\begin{tabular}{lccc}
\hline \multirow{2}{*}{ Variables investigated } & \multicolumn{3}{c}{ Levels of each variable } \\
\cline { 2 - 4 } & $\mathbf{1}$ & $\mathbf{2}$ & $\mathbf{3}$ \\
\hline A: ratio of berbamine to chitosan $(\mathrm{w} / \mathrm{w})$ & $1: 5$ & $1: 10$ & $1: 15$ \\
B: percentage of emulsifier $(\operatorname{span} 80, \mathrm{v} / \mathrm{v})$ & $2 \%$ & $4 \%$ & $6 \%$ \\
C: volume of glutaraldehyde $(\mathrm{mL})$ & 1 & 2 & 3 \\
D: reaction temperature $\left({ }^{\circ} \mathrm{C}\right)$ & 60 & 70 & 80 \\
\hline
\end{tabular}


of the cross-linked framework (as a result of the reaction between the - $\mathrm{CHO}$ groups of glutaraldehyde and the $-\mathrm{NH}_{2}$ groups of chitosan) is easily destroyed in phosphate buffer solution ( $\mathrm{pH}$ 7.4) resulting in more space for water within the matrix of the microspheres. In phosphate buffer solution ( $\mathrm{pH}$ 7.4) and acid condition ( $\mathrm{pH} 1.2)$, the microspheres reached swelling equilibrium at approximately 8 and 10 hours, respectively.

\section{Fourier transform infrared spectroscopy}

Owing to each specific chemical bond often has a unique energy absorption, FTIR spectroscopy has been extensively applied to identify the presence of certain functional groups or chemical bonds in a molecule. Figure 2 shows FTIR spectra of chitosan, agarose, berbamine, blank chitosan-agarose microspheres and berbamine loaded chitosan-agarose microspheres. The absorption bands at 1658 and $1600 \mathrm{~cm}^{-1}$ were ascribed to $\mathrm{N}-\mathrm{H}$ bending vibrations in the primary amine of chitosan (Figure $2 a)^{[14]}$, but they were weaken in chitosan-agarose microspheres and the new absorption band at $1650 \mathrm{~cm}^{-1}$ appeared (Figure 2d), which could be attributed to the interaction between the - $\mathrm{CHO}$ groups of glutaraldehyde and the $-\mathrm{NH}_{2}$ groups of chitosan in microspheres ${ }^{[15]}$. As shown in Figure $2 \mathrm{~b}$, the characteristic absorption bands of agarose were observed at $1076 \mathrm{~cm}^{-1}$ (C-O, axial deformation), $933 \mathrm{~cm}^{-1}$ (characteristic of 3, 6-anhydrogalactose) and $889 \mathrm{~cm}^{-1}$ (attributed to $\mathrm{C}-\mathrm{H}$ angular deformation of anomeric carbon) ${ }^{[16]}$. Pure berbamine spectra showed the characteristic absorption bands at 1515, 1275 and $1117 \mathrm{~cm}^{-1}$ (Figure 2c) ${ }^{[17]}$, which also appeared in the spectra of berbamine loaded chitosan-agarose microspheres (Figure 2e) at the same wavenumbers suggesting no interactions between the drug and the carrier. These results indicated that berbamine had been successfully loaded in the chitosan-agarose microspheres by physical entrapment.

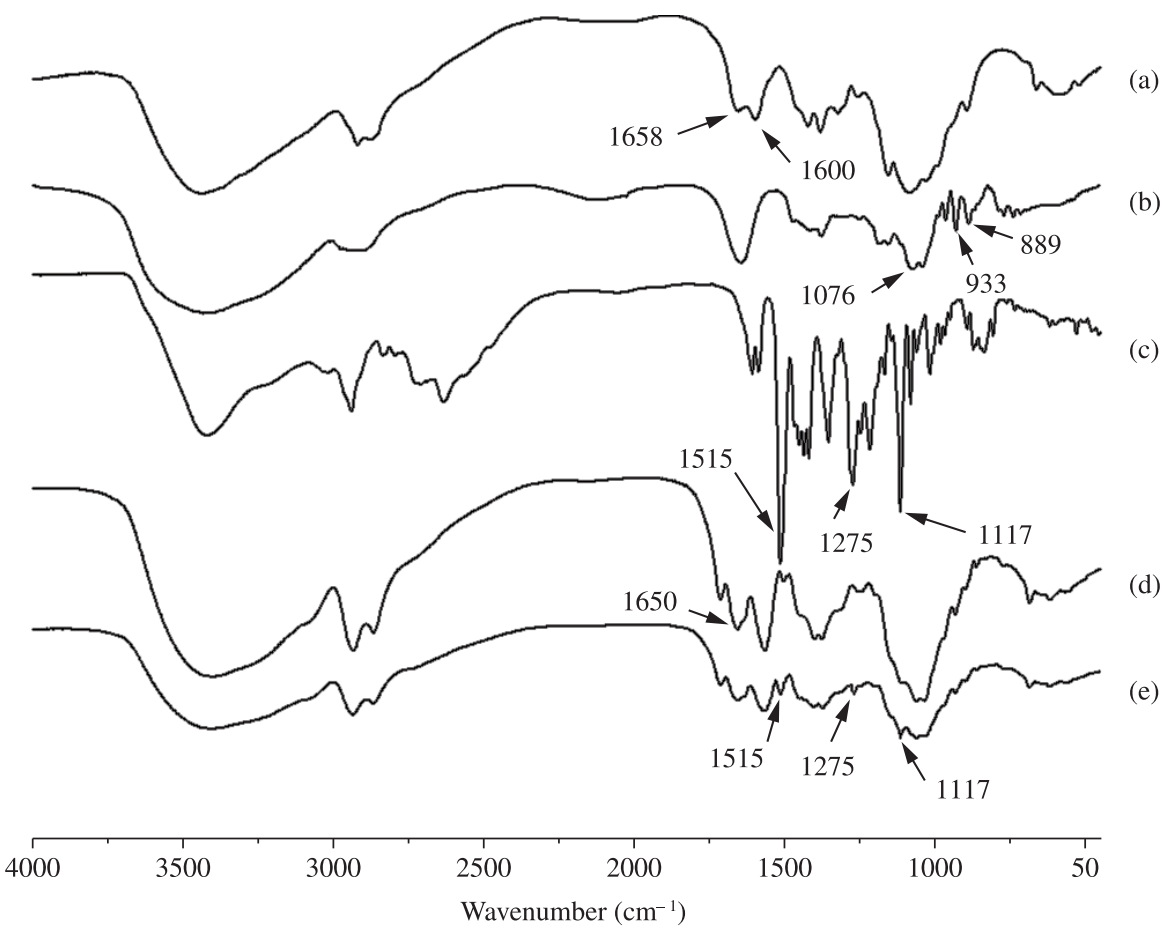

Figure 2. Infrared spectra of chitosan (a), agarose (b), berbamine (c), blank chitosan-agarose microspheres (d) and berbamine loaded chitosan-agarose microspheres (e).

Table 2. Experimental arrangement and test results.

\begin{tabular}{|c|c|c|c|c|c|c|c|}
\hline \multirow{2}{*}{$\begin{array}{c}\text { Experiment } \\
\text { number }\end{array}$} & \multicolumn{4}{|c|}{ Variables investigated } & \multirow{2}{*}{ EE \% } & \multirow{2}{*}{ LC\% } & \multirow{2}{*}{$\mathrm{QI}(\mathrm{EE} \%+\mathrm{LC} \%)$} \\
\hline & $\mathbf{A}$ & B & $\mathbf{C}$ & D & & & \\
\hline 1 & 1 & 1 & 1 & 1 & 12.42 & 3.50 & 15.92 \\
\hline 2 & 1 & 2 & 2 & 2 & 83.71 & 8.24 & 91.95 \\
\hline 3 & 1 & 3 & 3 & 3 & 48.33 & 6.52 & 54.85 \\
\hline 4 & 2 & 1 & 2 & 3 & 77.62 & 7.68 & 85.30 \\
\hline 5 & 2 & 2 & 3 & 1 & 53.27 & 5.52 & 58.79 \\
\hline 6 & 2 & 3 & 1 & 2 & 61.36 & 6.70 & 68.06 \\
\hline 7 & 3 & 1 & 3 & 2 & 44.29 & 4.26 & 48.55 \\
\hline 8 & 3 & 2 & 1 & 3 & 37.91 & 6.26 & 44.17 \\
\hline 9 & 3 & 3 & 2 & 1 & 68.27 & 6.18 & 74.45 \\
\hline $\mathrm{k}_{1}$ & 54.24 & 49.92 & 42.72 & 49.72 & & & \\
\hline $\mathrm{k}_{2}$ & 70.72 & 64.97 & 83.90 & 69.52 & & & \\
\hline $\mathrm{k}_{3}$ & 55.72 & 65.79 & 54.06 & 61.44 & & & \\
\hline $\mathrm{R}$ & 16.48 & 15.87 & 41.18 & 19.80 & & & \\
\hline
\end{tabular}




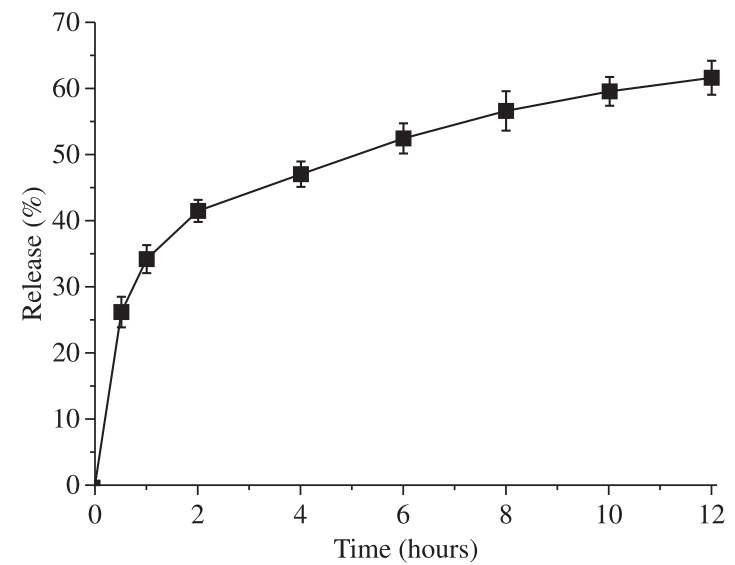

Figure 3. In vitro release profile of berbamine from microspheres.

Table 3. The applied release models.

\begin{tabular}{cc}
\hline Model & Equation \\
\hline Zero-order & $\mathrm{Q}_{\mathrm{t}}=\mathrm{Q}_{0}+\mathrm{K}_{0} \mathrm{t}$ \\
First-order & $\ln \mathrm{Q}_{\mathrm{t}}=\ln \mathrm{Q}_{0}+\mathrm{K}_{\mathrm{f}} \mathrm{t}$ \\
Higuchi & $\mathrm{Q}_{\mathrm{t}}=\mathrm{Q}_{0}+\mathrm{K}_{\mathrm{h}} \mathrm{t}^{1 / 2}$ \\
\hline
\end{tabular}

$\mathrm{Q}$, amounts of drug released in time $t ; \mathrm{Q}_{0}$, initial amounts of drug in the microspheres; $\mathrm{K}_{0}, \mathrm{~K}_{\mathrm{f}}, \mathrm{K}_{\mathrm{h}}$, release rate constants.

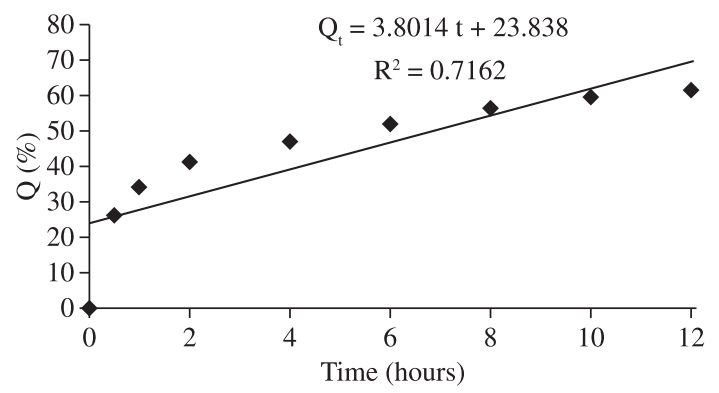

Figure 4. The fitting curve of zero order release equation.

\section{In vitro release of berbamine}

The cumulative percent of berbamine released from microspheres versus time plots were presented in Figure 3. It is obvious that in the early stages berbamine release in vitro exhibited a burst $(\approx 26 \%$ ) suggesting that few adsorbed drugs were soon released from the surface of microspheres. Then more than $60 \%$ berbamine was released from microspheres by 12 hours indicating that most berbamine drugs were included inside the cross-linked shell of microspheres. Apparently, it showed a sustained release pattern indicating the excellent nature of chitosan-agarose microspheres to release the drug.

Berbamine release kinetics were analyzed by various mathematical models, which were applied considering the amounts of drug released from 30 minutes to 12 hours. Table 3 gave the models tested and Figures 4-6 presented the results. Correlation and linear regression analyses were performed using Origin 7.5.

It can be seen that the step of drug penetrating through the chitosan-agarose microspheres is rate-limiting, therefore, the rate of drug release from microspheres is considered to be sustained. Considering the $\mathrm{R}^{2}$ values, the calculated zero-order model (Figure 4) and first-order model (Figure 5) failed to fit drug release from microspheres. As shown in Figure 6, the release kinetics may be square root time dependent, as in the Higuchi model, which can describe the drug release from microspheres. The microspheres enable drug continuous release over prolonged periods.

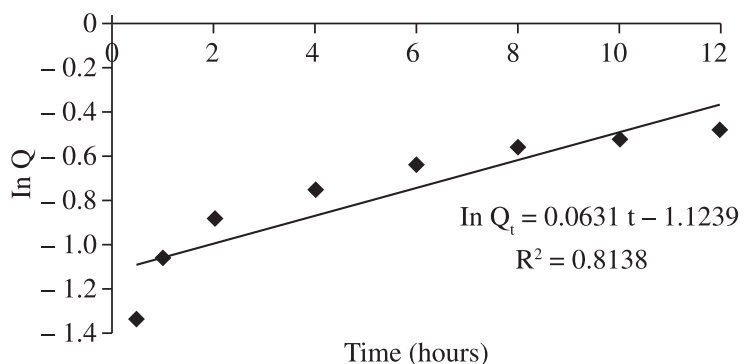

Figure 5. The fitting curve of first order release equation.

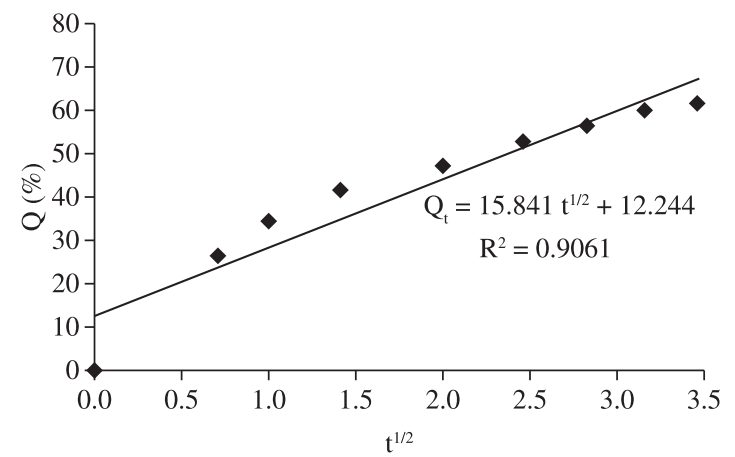

Figure 6. The fitting curve of Higuchi release equation.

\section{Conclusions}

We have successfully prepared the chitosan-agarose composite microspheres for berbamine delivery using emulsification method by orthogonal design. Encapsulation efficiency and loading capacity of microspheres were $84.57 \%$ and $8.44 \%$, respectively. Swelling test showed that the swelling ratio of microspheres at $\mathrm{pH} 7.4$ was higher than that at $\mathrm{pH}$ 1.2. FTIR indicated that berbamine had been successfully loaded in the chitosan-agarose microspheres by physical entrapment. In vitro studies demonstrated that berbamine was released from microspheres in a significantly sustained fashion.

\section{Acknowledgements}

This work was supported by the Program for Excellent Talents in Guangdong Ocean University (Grant no. 0912118).

\section{References}

1. Xu, R. Z.; Dong, Q. H.; Yu, Y.; Zhao, X.; Gan, X.; Wu, D.; Lu, Q.; Xu, X. \& Yu, X. F. - Leuk. Res., 30, p.17 (2006). PMid:16023722. http:// dx.doi.org/10.1016/j.leukres.2005.05.023

2. Wei, Y.; Xu, L.; Liang, Y.; Xu, X. \& Zhao, X. - Acta Pharmacol. Sin., 30, p.451 (2009). PMid:19270722. http://dx.doi.org/10.1038/aps.2009.19

3. Foster, N. \& Hirst, B. - Adv. Drug Deliver. Rev., 57, p.431 (2005). PMid:15560950. http://dx.doi.org/10.1016/j.addr.2004.09.009

4. Tamber, H.; Johansen, P.; Merkle, H. P. \& Gander, B. - Adv. Drug Deliver. Rev., 57, p.357 (2005). PMid:15560946. http://dx.doi. org/10.1016/j.addr.2004.09.002

5. Rinaudo, M. - Prog. Polym. Sci., 31, p.603 (2006). http://dx.doi. org/10.1016/j.progpolymsci.2006.06.001

6. Ravi Kumar, M. N. V.; Muzzarelli, R. A. A.; Muzzarelli, C.; Sashiwa, H. \& Domb, A. J. - Chem. Rev., 104, p.6017 (2004).

7. Qi, L. F.; Xu, Z. R.; Jiang, X.; Li, Y. \& Wang, M. Q. - Bioorg. Med. Chem. Lett., 15, p.1397 (2005). PMid:15713395. http://dx.doi. org/10.1016/j.bmcl.2005.01.010

8. Qi, L. F. \& Xu, Z. R. - Bioorg. Med. Chem. Lett., 16, p.4243 (2006). PMid:16759859. http://dx.doi.org/10.1016/j.bmcl.2006.05.078 
9. Sinha, V. R.; Singal, A. K.; Wadhawan, S.; Kaushik, R.; Kumria K.; Bansal, K. \& Dhawan, S. - Int. J. Pharmaceut., 274, p.1 (2004). PMid:15072779. http://dx.doi.org/10.1016/j.ijpharm.2003.12.026

10. Deszczynski, M.; Kasapis, S. \& Mitchelle, J. R. - Carbohyd. Polym., 53, p.85 (2003). http://dx.doi.org/10.1016/S0144-8617(02)00327-2

11. Lahooti, S. \& Sefton, M. V. - Biomaterials, 21, p.987 (2000). http:// dx.doi.org/10.1016/S0142-9612(99)00251-3

12. Watase, M.; Nishinari, K.; Clark, A. H. \& Ross-Murphy, S. B. - Macromolecules, 22, p.1196 (1989). http://dx.doi.org/10.1021/ ma00193a034

13. Xue, Z. X.; Yang, G. P.; Wang, G. C.; Niu, J. F. \& Cao, X. Y. - J. Appl. Polym. Sci., 103, p.2759 (2007). http://dx.doi.org/10.1002/app.25335
14. Lim, S. H. \& Hudson, S. M. - Carbohydr. Res., 339, p.313 (2004). PMid:14698889. http://dx.doi.org/10.1016/j.carres.2003.10.024

15. Çetinus, Ş. A.; Öztop, H. N. \& Saraydin, D. - Enzyme Microb. Tech., 41 p.447 (2007). http://dx.doi.org/10.1016/j.enzmictec.2007.03.014

16. Garcia, R. B. \& Vidal, R. R. L. - Polímeros, 10, p.155 (2000). http:// dx.doi.org/10.1590/S0104-14282000000300012

17. Liu, G. S.; Chen, B. Z.; Song, W. Z. \& Xiao, P. G. - Acta Botanica Sin., 20, p. 9 (1978).

Enviado: 08/10/11

Reenviado: 05/04/12

Aceito: $14 / 04 / 12$ 